\title{
A Research about the Application of Information Technology in the Precision Agriculture: Taking the Operating System of Shanghai Agriculture Economy as an Example
}

\author{
Hong Yao ${ }^{1,2}$ and Yong-xing $\mathrm{Wu}^{1, *}$ \\ ${ }^{1}$ College of Resources and Environmental Science, East China Normal University, \\ Shanghai, China \\ ${ }^{2}$ College of Ecology and Tourism, Shanghai Business School, Shanghai, China \\ juddy_yao@163.com
}

\begin{abstract}
In the background of the digital agriculture advanced step-by-step, Shanghai government is also accelerating the pace of agricultural informationization. "Precision Agriculture" has become a pointcut of "Digital Shanghai". Building the Operating System of Shanghai Agricultural Economy is a meaningful practice in Shanghai digital agriculture engineering. Its powerful function of spatial data analysis and inquiry provides the support system for the realization of "precision agriculture". It can provide the decision support and the information service for the government.
\end{abstract}

Keywords: precision agriculture, GIS, the Operating System of Shanghai Agricultural Economy.

\section{$1 \quad$ Introduction}

With the farm produce's large scale marketing and circulation coming into being, the future sustainable development of agriculture in Shanghai will, to a great extent, be decided by the restructuring and optimization of agriculture. On this background, Shanghai's agriculture has quickened the transformation from "Quantity Agriculture" to "Quality Agriculture", from planning typed into market-oriented, from suburban into modern urban agriculture. The government has accordingly adjusted its management, further stressing the macro adjustment and the function of service. The utilization of modern information technology enables the dynamic monitoring and timely appraisal of achievement and outcome of main information of Shanghai suburban agricultural economy operation, thus providing support for relevant government departments in their macro-control and management of agriculture in a timely, scientific and efficient

* Corresponding author. 
way. Also farmers can obtain effective guiding information in their optimization of their agriculture investment, reasonable restructuring, and gaining higher yield. Obviously the application of information technology in precision agriculture is one of important measures of Shanghai Municipal Government in realizing "Precision Agriculture" for the sustainable development of agriculture.

\section{The Definition of "Precision Agriculture"}

The concept of Precision Agriculture or Precision Farming was put forward by some U.S and Canadian agriculture research institutions in the 1990s. Precision Farming is a modern farming operation and management system, supported by the information technology, and exerting a series of modern farming techniques and managing expertise according to variations of locations and operated in a fixture of location, timing and quantity. The basic content of precision farming is to adjust the investment of crops according to the condition of the soil, in which farmers, after confirming the soil condition and the mutation of the space of productivity, decide the objective of the farming. And in this way, by "Systematic diagnosis, optimized recipe, technical installation and scientific management", farmers can fully utilize the fertility of the soil, thus obtaining higher yield with the least and most economical investment without damaging the environment and obtaining economic and environmental benefit by efficiently utilizing various agricultural resources. Ten systems are needed for the technical support in the realization of the precision farming which include the GPS, Information Collection System for Farmland, Farmland Remote Sensing and Monitoring System, Farm GIS, Agricultural Expert System, Smart Farming Equipment System, Environment Monitoring System, Integration System, Network Management System and Training System. The core of the system is the establishment of a complete farmland GIS. Precision Farming can be said as a brand-new farming with the complete integration of information technology and agricultural production, the goal of which is not only for achieving economic efficiency but also for social and ecologic benefits.

\section{The Present Situation and Problems in Precision Farming in Shanghai}

\subsection{The Present Situation}

Since the beginning of the 21st century, the agriculture in Shanghai has entered a new phase of development and the traditional suburban farming, which only provides farm produce, is no longer suitable for the status of Shanghai as internationalized metropolis. At present the agriculture of Shanghai is undertaking the transformation from being quantitative to qualitative, from suburban-style to metropolis, from extensive farming to integrated farming, from production-styled to service-oriented farming. The changes of farming functioning and ways of farming require urgently the change of mode of agricultural production and management and service system as well. Precision farm has 
become an inevitable option for Shanghai to develop its metropolitan agriculture because the information resources for agriculture in Shanghai have become multi-factored, with the characteristic of large quantity, effectiveness of timing and broadness of respects concerned. Further developing digital management of agriculture in Shanghai will have a profound sense for pushing forward precision agriculture in Shanghai with the advantages of Shanghai's human resources of GIS, software research and development and complete infrastructure of information technology.

\subsection{Advantages and Disadvantages}

Shanghai has the superior technical conditions and basis in realizing the precision agriculture. With speeding up construction of "digital city" and "digital Shanghai", Shanghai Municipal Agricultural Commission (SMAC) and Shanghai Municipal Agricultural and Forestry Bureau (MAFB) accelerate the construction of digital agriculture. They developed many practical works around the digital agriculture in recent years. First, the network publishing system of agricultural information has basically completed, including building the interior local area network platform of each agriculture department, developing the intranet information management system about the vegetable planting, animal husbandry and aquaculture, and including publishing all kinds of the agricultural information on web. Second, the basic research and development on sharing the basic data and technical standard has been launched. It includes the developer guide for the application system of Shanghai agricultural GIS, the agricultural information classification and coding standard in Shanghai agricultural GIS, working out the classification standard about agriculture industry in thematic mapping and the symbols norms. Third, some application projects have been carried out successively, including the dynamic monitoring system of non-point pollution control of farmland fertilizers and pesticides in Shanghai, the planning management information system of forestry resources in Shanghai, the GIS application system of animal husbandry management, etc. This series of practices on digital agriculture laid the foundation of the technology for achieving precision agriculture. In addition, there are some disadvantages in the construction of Shanghai precision farming. For example, the construction of network infrastructure is relatively inadequate in Shanghai suburbs. There are great differences on the Information infrastructure in different areas, lack of integrated talent in the agricultural information field. And all kinds of application systems of agricultural informationization also lack sharing mechanism or can't be integrated effectively. These problems in the development need to be considered urgently and should be solved gradually in the future process of development of Shanghai precision agriculture.

\section{The Relationship between "the Operating System of Shanghai Agricultural Economy" and "Precision Agriculture"}

The Operating System of shanghai Agricultural Economy is a practical project of Shanghai digital agriculture. It provides the support of technology and information for 
achieving precision agriculture. The system bases on GIS, database and the network technology, and has established the farming application systems facing the management, the decision-making, the relevant departments and specific users. Because the ordinary farmers are the major producers in suburban agriculture, the system takes advantage of progressive network GIS technology and regards the retail farmers as monitoring objects. In each plot the farmers should keep steady production. Among the plots there are objective differences. Thus, the system must follow these principles and realize digitalization in all aspects such as the agricultural production and circulation of the monitoring plots. This system constructed a dynamic professional database for the planting and economic operation. Through the comprehensive treatment and analysis, it can provide analysis and evaluation of the thematic information about the planting input-output and the dynamic monitoring data in Shanghai suburbs. It can also evaluate the operating performance of summer crops, autumn crops and crops for rotation under the different patterns, and express in space. The GIS expressive functions of spatial analysis, query and visualization not only show overall situation and spatial distribution pattern about the planting input, output and operating performance, but also show the production state of every monitoring point, various crops and every crop in different suburban, the structure of input and output, the operating performance, and the performance evaluation information in the conditions of different operating scale, different product certification and different market flow direction. All these provide scientific basis for the decision making of the agricultural department. The Operating System of Shanghai Agricultural Economy is a kind of management model of digital agriculture, which is line with the development foundation of crop production in Shanghai. After five years of operation, this system has already completed the construction and management of the dynamic monitoring network, remote data entry and the construction of professional database on the planting industry. It has exerted the certain function and guided the practical planting work. Thus, the Operating System of Shanghai Agricultural Economy is a meaningful practice in the huge digital agriculture projects of Shanghai, and provides the necessary conditions for realizing Shanghai precision agriculture.

\section{Technical Framework and Workflow of "the Operating System of Shanghai Agricultural Economy"}

\subsection{Technical Framework of the System}

The implementation of this system adopts the $\mathrm{B} / \mathrm{S}$ and $\mathrm{C} / \mathrm{S}$ architecture (as shown in Figure 1). It's comprised of the client (browsers), application service layer and the database server. All operation and data are deposited in server. Through the general Web browser the client can propose request of query and analysis. And the server returns the results. The client can realize the functions of data acquisition and editing, GIS inquire, statistical analysis and so on. The application service layer is the core of the system, is also a bridge between the client and the underlying database. The underlying database server includes the central database and model library. 


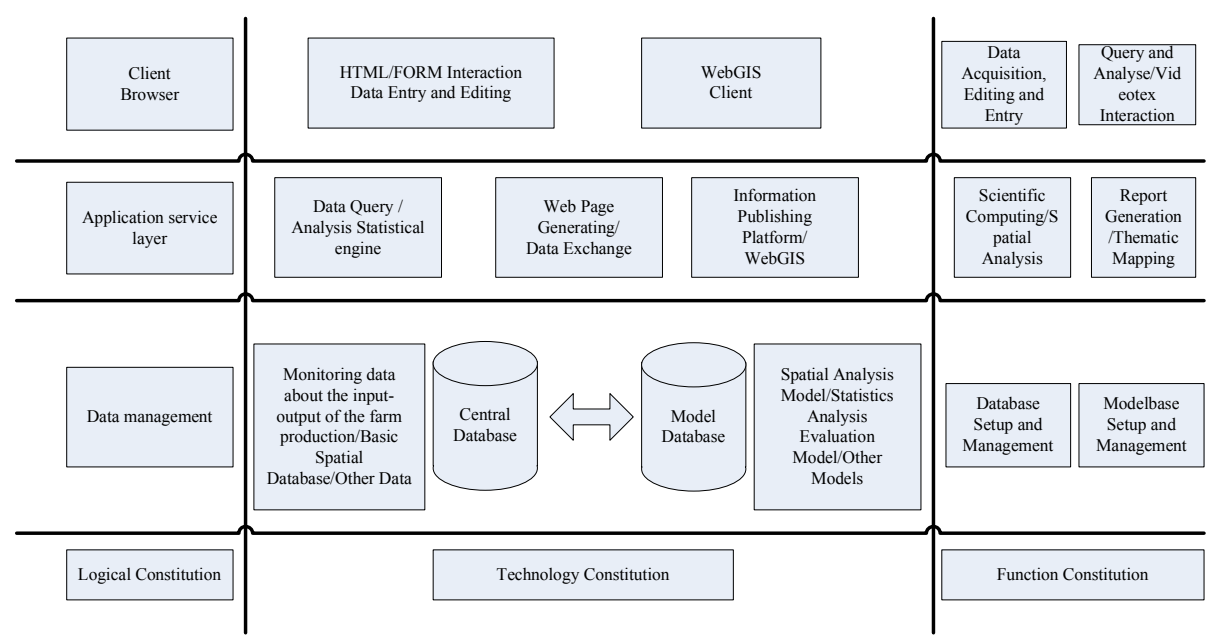

Fig. 1. The technical framework about "the Operating System of Shanghai Agricultural Economy"

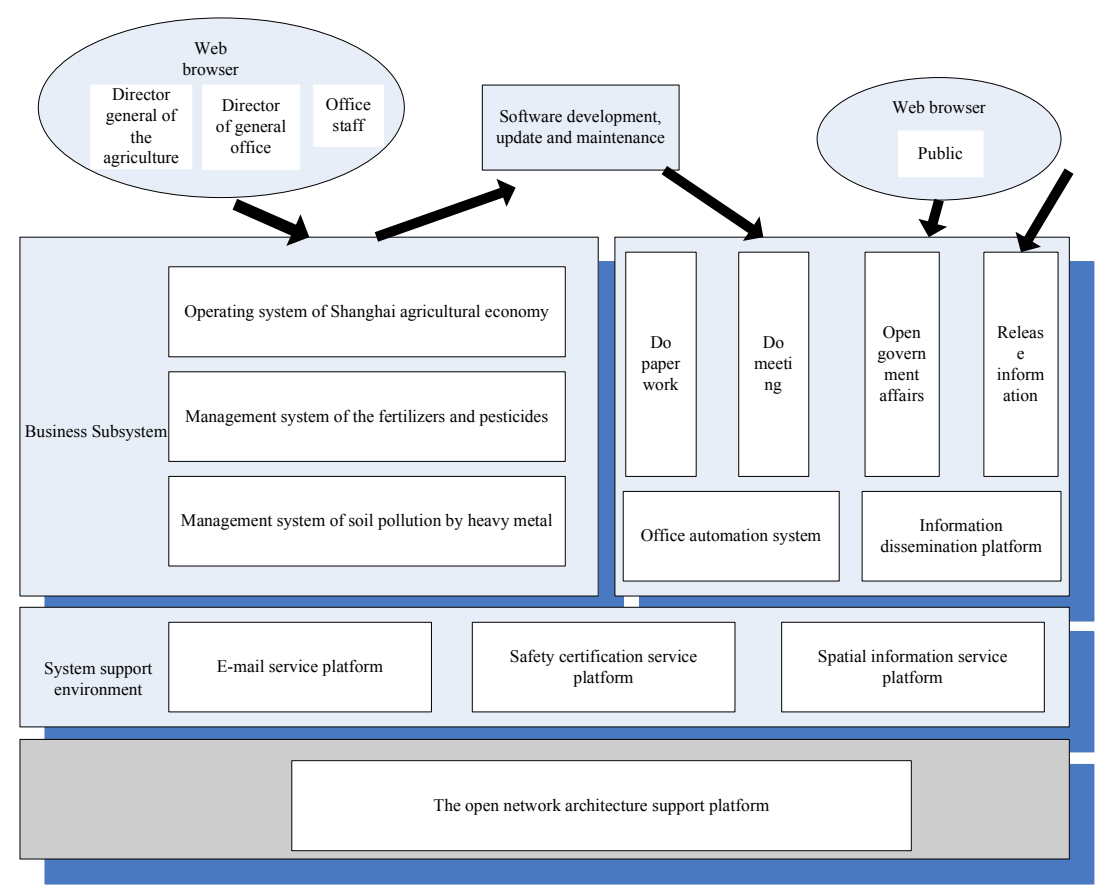

Fig. 2. The schematic diagram of "Shanghai Agricultural Comprehensive Management Information Platform" 
On the business logic, this system is an important component of comprehensive management information platform for Shanghai agriculture. The design of whole system based on an open system, fully considering the data statistics within the agriculture department and the data exchange with other relevant departments, and strived to achieve standardization.

GIS platform software of this system uses the technical route combining the independent development of GIS components with commercial GIS platform. The whole framework system conforms to the standard of TC211/OpenGIS, and can carry out interoperability and data exchange with other GIS software (such as ArcGIS, MapInfo, GeoMedia) that fits this standard.

\subsection{The Workflow of the System}

The research of whole project contains several tasks, including the system design, the questionnaire design, the data acquisition and database design, the data input, the model design, programming and debugging, drawing up the achievements, and documentation, etc.(as shown in Figure 3)

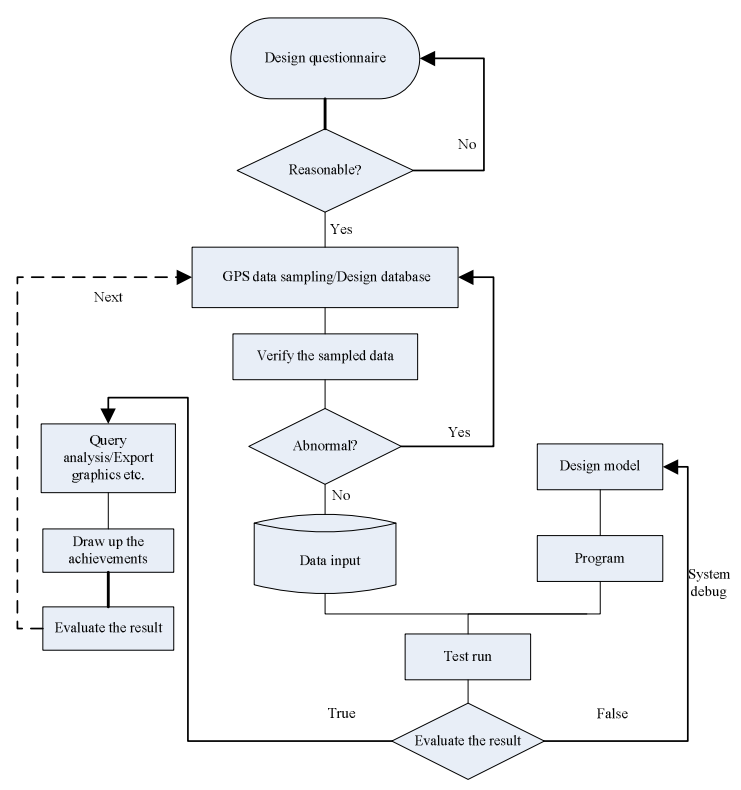

Fig. 3. The workflow of "the Operating System of Shanghai Agricultural Economy"

\section{Database Design of "the Operating System of Shanghai Agricultural Economy"}

The data of this system includes the attribute data, the spatial data and special graphics library data. The data storage and access use the technical framework of MSADO, and 
database system software adopts ORACLE, in order to make full use of the existing database system's functions in multi-user management, query and analysis, and safety and affairs management.

\subsection{Basic Space Database}

The spatial data accuracy is based on "the Shanghai Digital Agriculture Platform for Basic Data". And the administrative division level reaches the township. The coordinate system combined the coordinates system of WGS84 (the coordinates system of GPS) with Shanghai local coordinate system. The system supports two coordinate systems transformation and the real-time updating of the dynamic GPS monitoring points.

\subsection{Service Database}

According to the crop type, the crops for rotation and the administrative division, the agricultural specialized data and the related attribute data are classified and graded. It has formed a database system facing the dynamic monitoring and management of agricultural economy. The table design of the database and the relationship among the tables are shown in figure 4. The sampled data and tabulate data, in different years and quarters, are in a list. So this design is beneficial to the year to year comparisons.

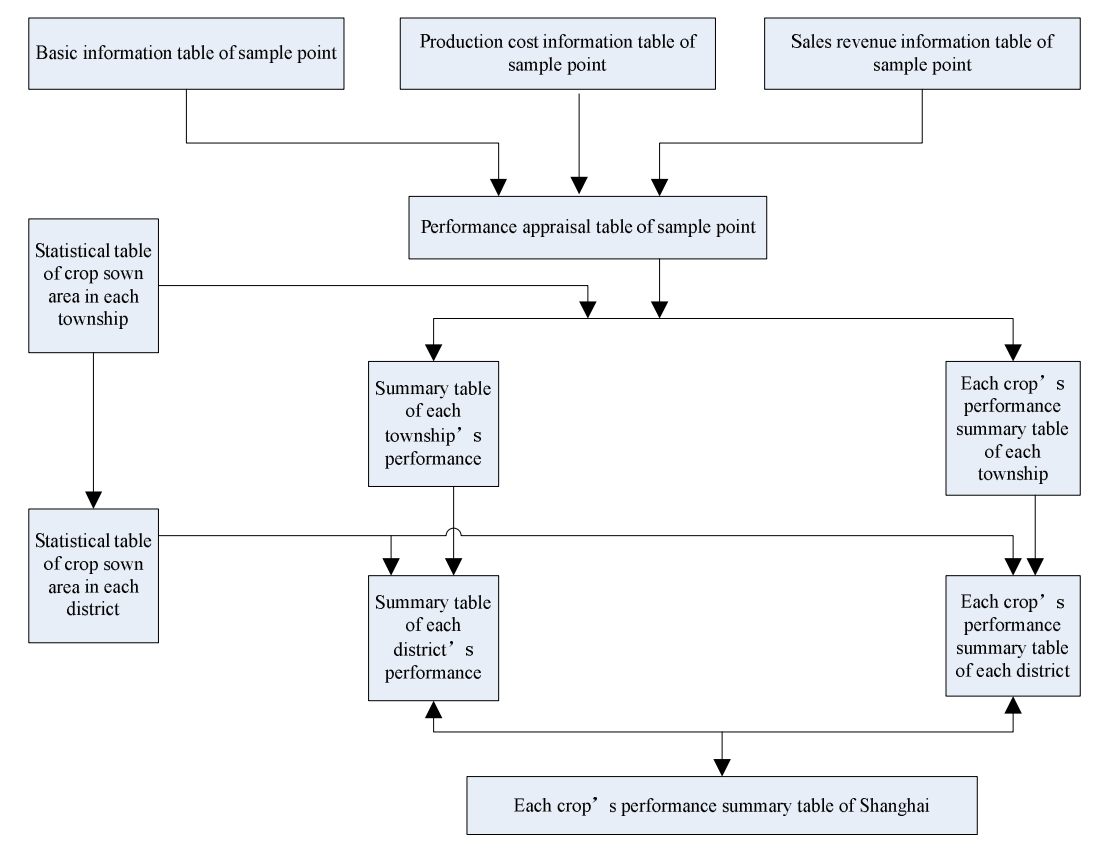

Fig. 4. Table design of the database and the relation among tables 


\subsection{Data Description}

\section{(1) Static data}

The static data in this system mainly includes basic geographical spatial database provided by the SMAC. It contains the, the road network and the channel network.

The data of the administrative division includes some basic layers of each district and township. It's used as the base map for the query, analysis and thematic mapping.

The data of the road network includes the layers about the main roads and streets in Shanghai. It can show the road network distribution in Shanghai suburban.

The data of the channel network includes the layers of the main rivers which are under municipal management, or under district management, or township management, etc. It's used for display the water system distribution in the suburban of Shanghai.

\section{(2) Dynamic data}

According to the farming production structure and layout features in Shanghai suburban, the expected dynamic trend, and the development characteristics of the planting industry in each county, we reasonably assigned and established the crops type and the crops for rotation of the dynamic monitoring points in the initial period. The system has formed the dynamic monitoring network which covered 121 townships of Shanghai, the main crops types, and the main crops for rotation types. Each township established 10 monitoring points. There are a total of 1210 monitoring points in Shanghai suburb. In the future, if the administrative division of the townships is adjusted, the monitoring points won't be changed in principle. If a monitoring point is revoked as a result of land acquisition and other reasons, we will add a monitoring point in the same township at the beginning of next year to maintain 1210 monitoring points. We have set up the field archive for every monitoring farmer (enterprise), timely recording the farming activities. So through the agricultural service system and agricultural office system of Shanghai, the archive has formed the standardized data acquisition system.

According to the farmers` demand and the system design, the questionnaire data of "the Operating System of Shanghai Agricultural Economy" mainly includes the following respects: the total cost of production, the output, the data of fertilizer and pesticides utilization, and the other relevant data. The total cost of production includes the material costs, the service costs, the labor costs, the other costs. The output includes the total quantity of production, the total of sale, the total value of production, and the total sales.

This system has completed the design of four basic attribute databases. One is the basic survey information database of 1210 monitoring points. The second database contains two basic sub databases which are the operating system of the summer crops and the operating system of the autumn crops. The third is the annual database characterized by the crops for rotation. The last is the grading summary database of the total sown area in Shanghai suburb. The attribute database takes the monitoring point numbers for the keyword to number, and carries out a targeted data survey of the economy operation for the monitoring farmers in the whole year. 


\section{The Goal of "the Operating System of Shanghai Agricultural Economy"}

(1) Taking use of GIS technology and based on "the Shanghai Digital Agriculture Platform for Basic Data", the system will complete establishing a dynamic professional database facing the planting production and economy operation.

(2) Through the system functions of the comprehensive processing and analysis, the system will provide the dynamic monitoring data of the planting input-output in Shanghai suburb, the process analysis and evaluation of the thematic information, the performance appraisal under the different conditions, and the spatial analysis and expression.

(3) Through the functions of GIS, such as the spatial analysis inquiry and visualization, the system could analyze and evaluate the situation, and show the spatial distribution characteristics about the input-output and performance of Shanghai planting production. Aiming at each monitoring point, each crop, or each district, it can also provide the performance evaluation information about the production situation, the constitution of the input-output, and the performance under the conditions of the different production scale, the product certification and the market flow direction. The system can provide the inquiry function of the related information.

(4) Through the GIS spatial analysis and inquiry of the system, we can know the application conditions of fertilizers and pesticides in every plot. So we can timely take measures to increase the utilizing efficiency of them in order to reduce the environmental pollution.

(5) The system has the ability to generate the relevant reports, the report forms, the statistical figure, and the thematic maps. And it supports the real-time inquires query and printout, the data exchange among the systems. These functions can provide the powerful decision support and information services for the macro-control and managing the planting production in the suburb of Shanghai.

In short, "the Operating System of Shanghai Agricultural Economy", by means of monitoring the huge data dynamically, gets the relevant information of the planting timely, and evaluates the real-time conditions of the planting. It is good for advancing the scientific and precision level of the crop production management, improving the economic benefit and social benefits and environmental benefits. And the system provides the technical support for achieving precision agriculture in Shanghai.

\section{References}

1. Wu, Y.-x., Li, W.-j., You, L.: On the construction of metropolitan agriculture in Shanghai. Geography And Territorial Research 16(3), 27-31 (2000) (in Chinese)

2. Han, J., Cai, Y., Cercone, N.: Data-driven discovery of quantitative rules in relational databases. IEEE Trans. Knowl. Data Eng. 5(1), 29-40 (1993)

3. Juan, W., Zha, L.-S.: Application of the Integration of GIS and SDM in Agriculture. Journal of Anhui Agricultural Sciences 36(29), 12974-12975, 12985 (2008) (in Chinese) 